HEP/123-qed

\title{
Polaron Self-trapping in a Honeycomb Net
}

\author{
Marco Zoli \\ Istituto Nazionale di Fisica della Materia - Universitá di Camerino, \\ 62032 Camerino, Italy.e-mail: zoli@campus.unicam.it
}

(November 21, 2018)

\begin{abstract}
Small polaron behavior in a two dimensional honeycomb net is studied by applying the strong coupling perturbative method to the Holstein molecular crystal model. We find that small optical polarons can be mobile also if the electrons are strongly coupled to the lattice. Before the polarons localize and become very heavy, there is infact a window of $e$-ph couplings in which the polarons are small and have masses of order $\simeq 5-50$ times the bare band mass according to the value of the adiabaticity parameter. The $2 \mathrm{D}$ honeycomb net favors the mobility of small optical polarons in comparison with the square lattice.
\end{abstract}

PACS: 63.10.+a, 63.20.Dj, 71.38.+i

Typeset using REVTEX 


\section{I.INTRODUCTION}

The transport properties of real systems are strongly affected by the presence of non linear potentials in the lattice [1] 3]. Nonlinearities may arise because of embedded impurities in the host lattice which favor trapping of the charge carriers [4] in any dimensionality [8] or, nonlinearities may be intrinsic to the system and driven by the electron-lattice interaction as assumed in the Molecular Crystal Model first proposed by Holstein [9] in the form of a discrete nonlinear Schrödinger equation for electrons coupled to harmonic phonons. Several theoretical methods have been developed in the last decades [11 [19] to analyse the ground state and finite temperature properties of the unit comprising the electron plus the surrounding local lattice deformation, namely the polaron. Central to these investigations is the concept of self-trapping traditionally denoting a transition between an infinite radius state at weak electron-phonon coupling and a finite radius polaron at strong e-ph coupling. The narrowing of the polaron band and the abrupt increase of the polaron effective mass versus e-ph coupling are the classical and related indicators of the transition event which may occur or not according to the degree of adiabaticity and the dimensionality of the system. When the characteristic phonon energy $\bar{\omega}$ becomes larger than the electronic bandwidth the antiadiabatic regime is attained. In this case it is generally accepted that the polaron wave function spreads over a few lattice sites (small polaron) with the polaron mass being a smooth function of the e-ph coupling. Instead, the polaron self-traps in the adiabatic regime and there is growing evidence that this event takes place in any dimensionality [15, 19,20].

When the lattice polarizations is confined to one or a few unit cells the carrier feels the details of the local structure: recent generalizations of the Holstein model have shown that the inclusion of on site lattice anharmonicity can substantially modify the size [21] and the mass 222 of the polaronic quasiparticle. While these findings could contribute to locate with more accuracy the self-trapping event in parameter space it is still unclear whether and how the transition depends on the lattice structure. To address this problem we focus here on the polaron mass renormalization in a two dimensional honeycomb net which can be viewed as 
a triangular Bravais lattice with a bases of diatomic molecules at the vertexes. The Holstein model is briefly reviewed and the results are discussed in Section II. The conclusions are drawn in Section III.

\section{THE HOLSTEIN MODEL HAMILTONIAN}

The Hamiltonian for the single electron in the Holstein model reads

$$
H=-t \sum_{i \neq j} c_{i}^{\dagger} c_{j}+g \sum_{i} c_{i}^{\dagger} c_{i}\left(a_{i}+a_{i}^{\dagger}\right)+\sum_{\mathbf{k}} \omega_{\mathbf{k}} a_{\mathbf{k}}^{\dagger} a_{\mathbf{k}}
$$

where the dimension dependence explicitly appears in the momentum space Hamiltonian

for the harmonic lattice vibrations. $c_{i}^{\dagger}\left(c_{i}\right)$ creates (destroys) a tight binding electron at the $i$ site and $t$ is the first neighbor hopping integral related to the bare electron half bandwidth $D$ by $D=z t, z$ being the coordination number. $a_{\mathbf{k}}^{\dagger}\left(a_{\mathbf{k}}\right)$ creates (destroys) a $\mathbf{k}$-phonon with frequency $\omega_{\mathbf{k}} \cdot g$ is the overall electron-phonon coupling constant.

In the strong coupling regime the Lang-Firsov approach [23] is reliable 24,25] and the polaron mass $m^{*}$ can be obtained via perturbative method. In $d$ dimensions the ratio between $m^{*}$ and the bare band mass $m_{0}$ is [20]:

$$
\begin{aligned}
\left(\frac{m^{*}}{m_{0}}\right)_{d} & =\frac{\exp \left(\bar{g}^{2}\right)}{1+z^{2} \operatorname{texp}\left(-\bar{g}^{2}\right) f\left(\bar{g}^{2}\right) /(\hbar \bar{\omega})} \\
\bar{g}^{2} & =\frac{2 g^{2}}{N} \sum_{k_{x}} \sin ^{2} \frac{k_{x}}{2} \sum_{k_{x}, k_{y}}\left(\hbar \omega_{\mathbf{k}}\right)^{-2} \\
f\left(\bar{g}^{2}\right) & =\sum_{s=1}^{+\infty} \frac{\left(\bar{g}^{2}\right)^{s}}{s s !}
\end{aligned}
$$

The series expansion in the last of eqs.(2) reflects the fact that the second order polaron self energy comprises the emission and absorption of an arbitrary number of phonons hence it is a sum over an infinite number of diagrams each having $s$ phonons between the two interaction vertexes. The second order of perturbative theory also introduces the effect of the adiabaticity parameter $z t / \hbar \bar{\omega}$ on $m^{*}$ which, in general, depends on dimensionality through: i) $g^{2} \propto d$, ii) the first neighbors number $z$, iii) the Brillouin zone sums and iv) the features of the phonon spectrum. 
We take a 2D honeycomb net equivalent to a triangular lattice with a two points basis. Each lattice site is a diatomic molecule with coordination number $z=6$. Hence, the phonon spectrum has both acoustical and optical branches whose analytical expressions can be deduced by a force constants parametrization scheme:

$$
\begin{aligned}
\omega^{2}\left(k_{x}, k_{y}\right) & =\frac{\beta+3 \gamma}{M} \pm \frac{1}{M} \cdot \sqrt{\beta^{2}+\gamma^{2} H\left(k_{x}, k_{y}\right)+\beta \gamma G\left(k_{x}, k_{y}\right)} \\
H\left(k_{x}, k_{y}\right) & =3+2\left(c_{x} c_{y}+s_{x} s_{y}+c_{3 x} c_{y}+s_{3 x} s_{y}+c_{2 x}\right) \\
G\left(k_{x}, k_{y}\right) & =2\left(2 c_{x} c_{y}+c_{2 x}\right)
\end{aligned}
$$

with: $c_{x}=\cos \left(k_{x} \sqrt{3} a / 2\right), c_{y}=\cos \left(k_{y} 3 a / 2\right), c_{2 x}=\cos \left(k_{x} \sqrt{3} a\right), c_{3 x}=\cos \left(k_{x} 3 \sqrt{3} a / 2\right)$, $s_{x}=\sin \left(k_{x} \sqrt{3} a / 2\right), s_{y}=\sin \left(k_{y} 3 a / 2\right), s_{3 x}=\sin \left(k_{x} 3 \sqrt{3} a / 2\right) . a$ is the lattice constant and $M$ is the reduced molecular mass. $\beta$ and $\gamma$ are the intra- and inter-molecular force constants respectively in terms of which one defines: $\omega_{0}^{2}=2 \beta / M, \omega_{1}^{2}=\gamma / M$ and the zone center optical frequency $\bar{\omega}=\sqrt{\omega_{0}^{2}+z \omega_{1}^{2}}$.

Previous investigations of the Holstein Hamiltonian [26] have shown that the intermolecular forces have to be sufficiently strong in order to predict the correct polaron bandwidth trend versus dimensionality. Thus the ground state properties of the Holstein model essentially depend on the strength of $\omega_{1}$ which should be of order $\simeq \omega_{0} / 2$. When this condition is fulfilled the polaron mass turns out to be substantially dimension independent. Larger $\omega_{1}$ values are admitted in the model although they encounter the obvious upper bound $\omega_{1} \leq \omega_{0}$ in a molecular lattice. This result (which has been proven in a large portion of parameter space ranging from fully adiabatic to antiadiabatic conditions) introduces a novel feature in the polaron landscape corroborated by Monte Carlo simulations [27], density matrix renormalization-group studies [15] and variational approaches.

Two quantities play a central role in polaron theory. The first, defined by

$$
\lambda=\frac{N g^{2}}{D \sum_{\mathbf{k}} \hbar \omega_{\mathbf{k}}}
$$

$N$ being the number of molecular sites, represents the ratio [28] between the polaron binding energy and the electronic half bandwidth. It yields the energetical gain due to small polaron 
formation with respect to the bare electronic state. The second, defined by

$$
\alpha=\frac{N g}{\sum_{\mathbf{k}} \hbar \omega_{\mathbf{k}}}
$$

, measures the lattice deformation associated with the quasiparticle formation. While in adiabatic systems the condition $\lambda>1$ signals the existence of the small polaron state, in antiadiabatic systems $\alpha>1$ is a more restrictive condition for small polaron formation 29.

Recent analysis [30 32] on the mobility of small polarons also in conjunction with models on polaronic high $T_{c}$ superconductivity [33 35] have led to reconsider the concept of selftrapped state which, although being intimately related to the small size of the quasiparticle, is not synonymous with small polaron state. Thus, if the narrowing of the polaron bandwidth (induced by an increasing e-ph coupling) marks the onset of the transition between large and small states still there is a range of $g$ values for which the polaron, although spread over a few lattice sites only, is not trapped and retains mobility properties. The self-trapping event can be instead associated with a rapid but continuos effective mass increase which is precisely located by looking at the curvature of the logarithm of the effective mass versus $g$. In our view this method, beyond embodying the full physical significance of the transition process, offers a simple criterion to select a "critical $g$ value" as an inflection point either in the logarithm of the effective mass or in its first derivative. While the former case would identify the point of most rapid increase of the effective mass, the occurence of the latter case distinguishes a peculiar point in the mass versus $g$ plot although the concavity-convexity change is absent.

In Fig.1(a), four plots of the polaron mass (in units of the bare band mass) as a function of the $g$ coupling are reported on while the corresponding curvatures of the logarithm of the mass are shown in Fig.1(b). High optical phonon frequencies are assumed. Our selected plots range from an extreme adiabatic $(t=200 \mathrm{meV})$ to a moderately antiadiabatic $(t=10 \mathrm{meV})$ regime. At a fixed $g$ the antiadiabatic polaron is always heavier than the adiabatic polarons but the peculiar points, resolved as the minima of the curves displayed in Fig.1(b), lie at decreasing $g$ values by decreasing the degree of adiabaticity. Then, an antiadiabatic polaron 
may self-trap already at $g \simeq 2.7$ weighing $m_{\text {eff }} \simeq 14$ while an extreme adiabatic polaron self-traps only at $g \simeq 3.8$ with $m_{\text {eff }} \simeq 123$. The correctness of our perturbative approach is monitored by the $\lambda$ and $\alpha$ values which are larger than one in all the displayed points consistently with the assumption on the existence of small polarons. In the intermediate adiabatic cases $t=100 \mathrm{meV}$ and $t=50 \mathrm{meV}$ we find $m_{e f f} \simeq 73$ at $g \simeq 3.5$ and $m_{e f f} \simeq 40$ at $g \simeq 3.3$ respectively. Thus, a $2 \mathrm{D}$ honeycomb net seems to sustain mobile adiabatic polarons in a window of strong coupling regimes approximately defined by $2.5 \leq g \leq 3.3$. In Figs.2, the phonon frequencies are still high, although much reduced with respect to Figs.1. As a main effect the polaron masses are roughly doubled while the peculiar minima of the second derivative of the logarithm of the effective mass (Fig.2(b)) do not shift substantially versus $g$ with respect to the corresponding cases in Fig.1(b). To emphasize the role of the lattice structure we have compared the adiabatic polaron (with $t=100 \mathrm{meV}$ ) in the present 2D honeycomb net with the previously investigated [20] square lattice. As an example, provided that: i) the same overall e-ph coupling $g=3.38$ is taken, ii) the same values of intra- and inter-molecular force constants are assumed $\left(\omega_{0}=50 \mathrm{meV}\right.$ and $\left.\omega_{1}=25 \mathrm{meV}\right)$ and these values are consistent with the strong coupling perturbative method, we find $m_{e f f} \simeq 140$ for the honeycomb net polaron against $m_{\text {eff }} \simeq 1200$ for the square lattice. Also the intermediate adiabatic polaron $(t=50 \mathrm{meV})$ behaves in a similar manner being $m_{\text {eff }} \simeq 200$ in the honeycomb net polaron against $m_{\text {eff }} \simeq 1700$ in the square lattice assuming the same input parameters as above. We have also considered the effect of the acoustical branch of the phonon spectrum on the polaron properties. In all cases, with different degree of adiabaticity, the minima of the second derivative (Fig.3(b)) occur at much lower $g$ values than for optical polarons. However, no physical meaning can be attached to these values since they are well outside the range of validity of the Lang-Firsov based perturbative method. When the method holds $(g>2.5)$ no peculiar point can be resolved in the polaron behavior versus $g$ which is anyway characterized by a huge mass renormalization. Figs.(3) have been reported also to point out that the occurence of distinctive features in the mass or mass derivative curves, far from being a cogent criterion for the self-trapping event, just indicates a trend 
which needs to be corroborated by the analysis of other independent quantities. 


\section{FIGURES}

FIG. 1. (a) Polaron masses (in units of bare band electron mass) versus $g$ in adiabatic and

antiadiabatic regimes with high frequency optical phonons: $\bar{\omega}=158 \mathrm{meV}$. (b) Second derivative of the logarithm of the effective mass with respect to $g$. 
FIG. 2. (a) Polaron masses (in units of bare band electron mass) versus $g$ in adiabatic and antiadiabatic regimes. The characteristic frequency of the optical phonons is: $\bar{\omega}=79 \mathrm{meV}$. (b) Second derivative of the logarithm of the effective mass with respect to $g$. 
FIG. 3. (a) Polaron masses (in units of bare band electron mass) versus $g$ in three adiabatic regimes. The electron couples here to the acoustical phonon branch. (b) Second derivative of the logarithm of the effective mass with respect to $g$.

\section{CONCLUSIONS}

We have developed a perturbative study of the molecular crystal model assuming the existence of strong electron-phonon coupling conditions which favor the formation of small polarons. The Lang-Firsov method permits to calculate the mass renormalization for specific structures once the phonon spectrum is known. Rather than applying the model to real systems as previously done for simple lattices, we have examined whether alternative structures as the 2D honeycomb net may host polarons which are both small and mobile. Infact we have found that adiabatic small polarons are lighter by roughly a factor eight than in the 
square lattice once the same input parameters are assumed. Adiabatic polarons have been studied as a function of the e-ph coupling and distinctive points in the mass behavior (versus $g$ ) have been selected and put in relation with a possible occurence of the self-trapping event. Although not ultimative this criterion seems plausible provided that such "self-trapping $g$ points" are obviously well within the range of applicability of the Lang-Firsov scheme. We find that small optical polarons in the honeycomb net can self-trap if the electron-lattice system couples in the range $3<g<4$. The exact location of the transition depends on the adiabaticity parameter with intermediate adiabatic polarons lying in the lower portion of that range. Our results suggest that adiabatic optical polarons can be mobile in the honeycomb net although a strong coupling regime holds. The small polaron effective mass is of order $\simeq 5-50$ times the bare band mass before the self-trapping point is attained, with more adiabatic polarons being lighter once the $e$-ph coupling is fixed.

This work has been done in Trequanda (Siena). 


\section{REFERENCES}

[1] Mott N F, 1987 Conduction in Non-Crystalline Materials Oxford:Clarendon

[2] Heeger A J, Kivelson S, Schrieffer J R, Su W -P, 1988 Rev. Mod. Phys. 60781

[3] Devreese J T, 1996 Polarons in Encyclopedia of Applied Physics (VCH Publishers, NY) 14383

[4] Wipf H, Magerl A, Shapiro S M, Satija S K, Thimlinson W, 1981 Phys. Rev. Lett. 46 947

[5] Scharf R, Bishop A R, 1991 Phys. Rev. A 436535

[6] Molina M I, Tsironis G P, 1993 Phys. Rev. B 4715330

[7] Gupta B C, Lee S B, 2001 Phys. Rev. B 63144302

[8] Dunlap D H, Kenkre V M, Reineker P, 1993 Phys. Rev. B 4714842

[9] Holstein T, 1959 Ann. Phys. (N.Y.) 8 325; ibid. 8343

[10] Toyozawa Y, 1961 Prog. Theor. Phys. 2629

[11] Emin D, Holstein T, 1976 Phys. Rev. Lett. 36323

[12] De Raedt H, Lagendijk A, 1983 Phys. Rev. B 27 6097; ibid., 1984301671

[13] Kopidakis G, Soukoulis C M, Economou E N, 1995 Phys. Rev. B 5115038

[14] La Magna A, Pucci R, 1996 Phys.Rev.B 538449

[15] Jeckelmann E, White S R, 1998 Phys. Rev. B 576376

[16] de Mello E V, Ranninger J, 1998 Phys. Rev. B 589098

[17] Robin J M, 1998 Phys. Rev. B 5814335

[18] Alexandrov A S, Kornilovitch P E, 1999 Phys. Rev. Lett. 82807 
[19] Romero A H, Brown D W, Lindenberg K, 1999 Phys. Rev. B 59 13728; ibid.,60 14080

[20] Zoli M, 2000 Phys. Rev. B 6114523

[21] Zolotaryuk Y, Christiansen P L, Rasmussen J J, 1998 Phys. Rev. B 5814305

[22] Voulgarakis N K, Tsironis G P, 2001 Phys. Rev. B 6314302

[23] Lang I J, Firsov Y A, 1963 Sov. Phys. JETP 161301

[24] Gogolin A A, 1981 Phys. Stat. Sol. (b)103 397

[25] Firsov Y A, Kabanov V V, Kudinov E K, Alexandrov A S, 1999 Phys. Rev. B 5912132

[26] Zoli M, 1998 Phys. Rev. B 5710555

[27] Kornilovitch P E, Pike E R, 1997 Phys. Rev. B 55 R8634

[28] Zoli M, 1999 Physica C 32471

[29] Zoli M, 2000 J.Phys.:Condens.Matter 122783

[30] Farias G A, da Costa W B, Peeters F M, 1996 Phys. Rev. B 5412835

[31] Emin D, 1996 Phys. Rev. B 531260

[32] de Mello E V, Ranninger J, 1997 Phys. Rev. B 55 14872; ibid., 19995912135

[33] Alexandrov A S, Mott N F, 1994 Rep. Prog. Phys. 571197

[34] Mustre de Leon J, Batistić I, Bishop A R, Conradson S D, Trugman S A, 1992 Phys. Rev. Lett. 683236

[35] Fehske H, Röder H, Wellein G, Mistriotis A, 1995 Phys. Rev. B 5116582 


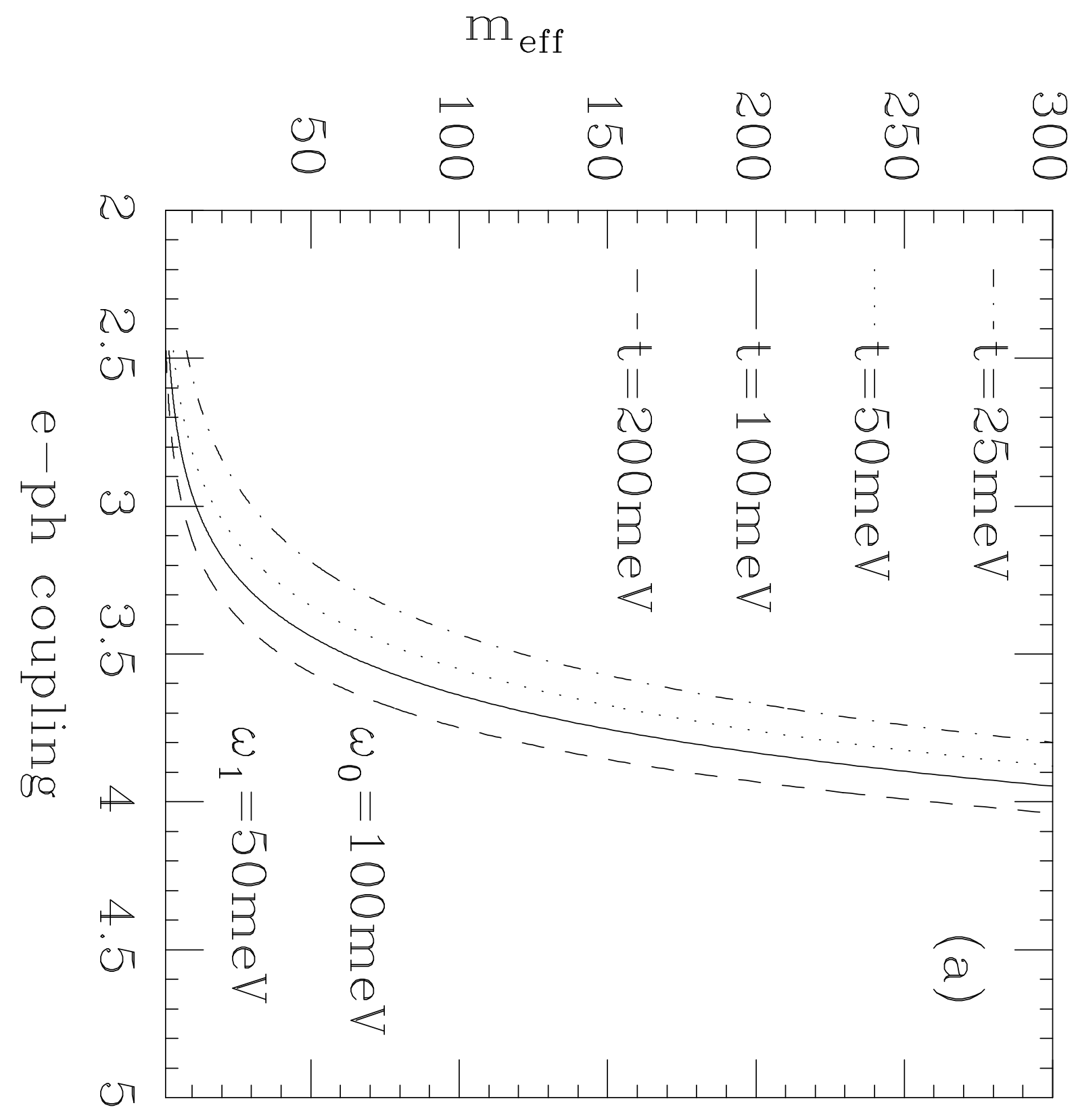




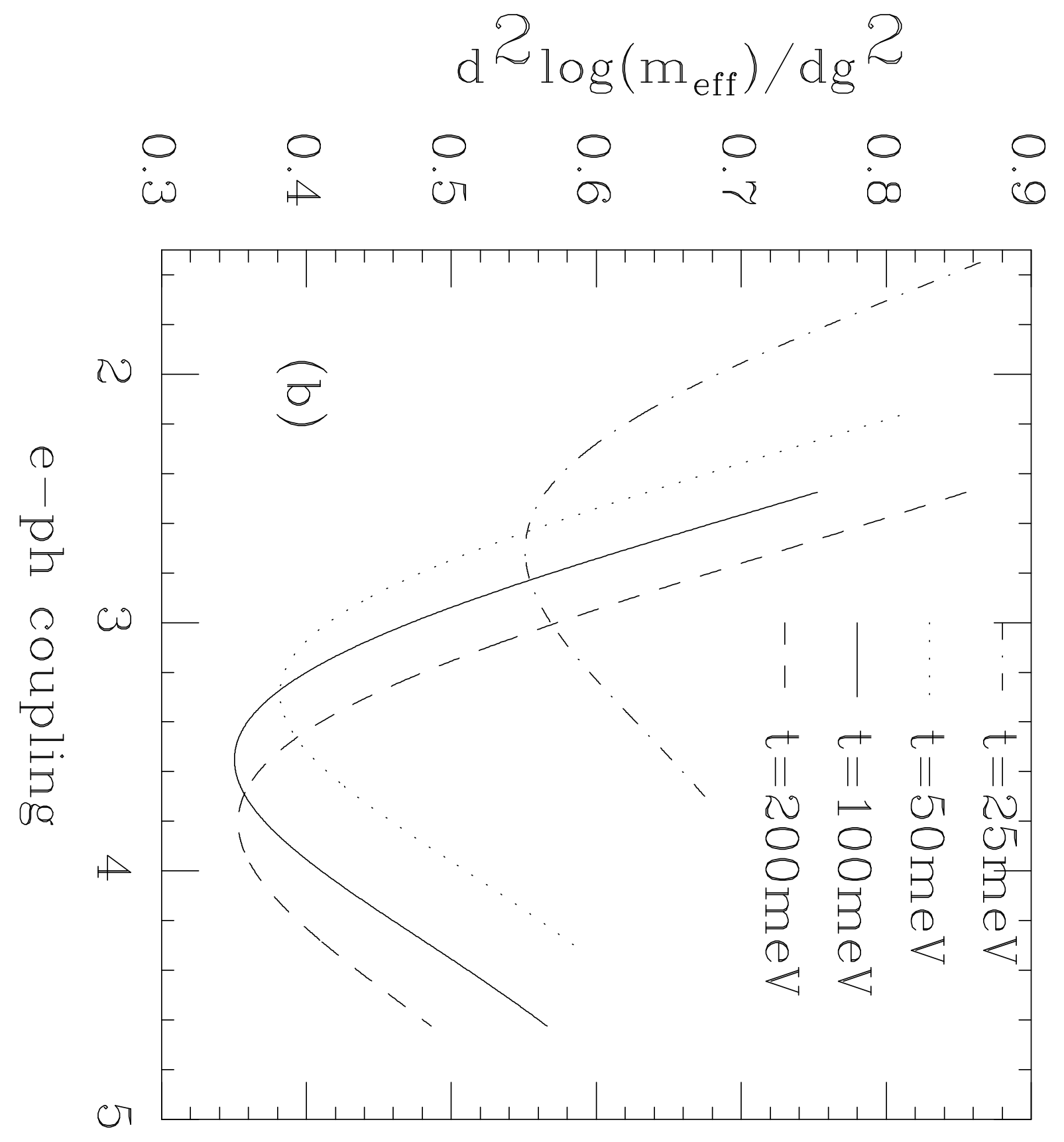




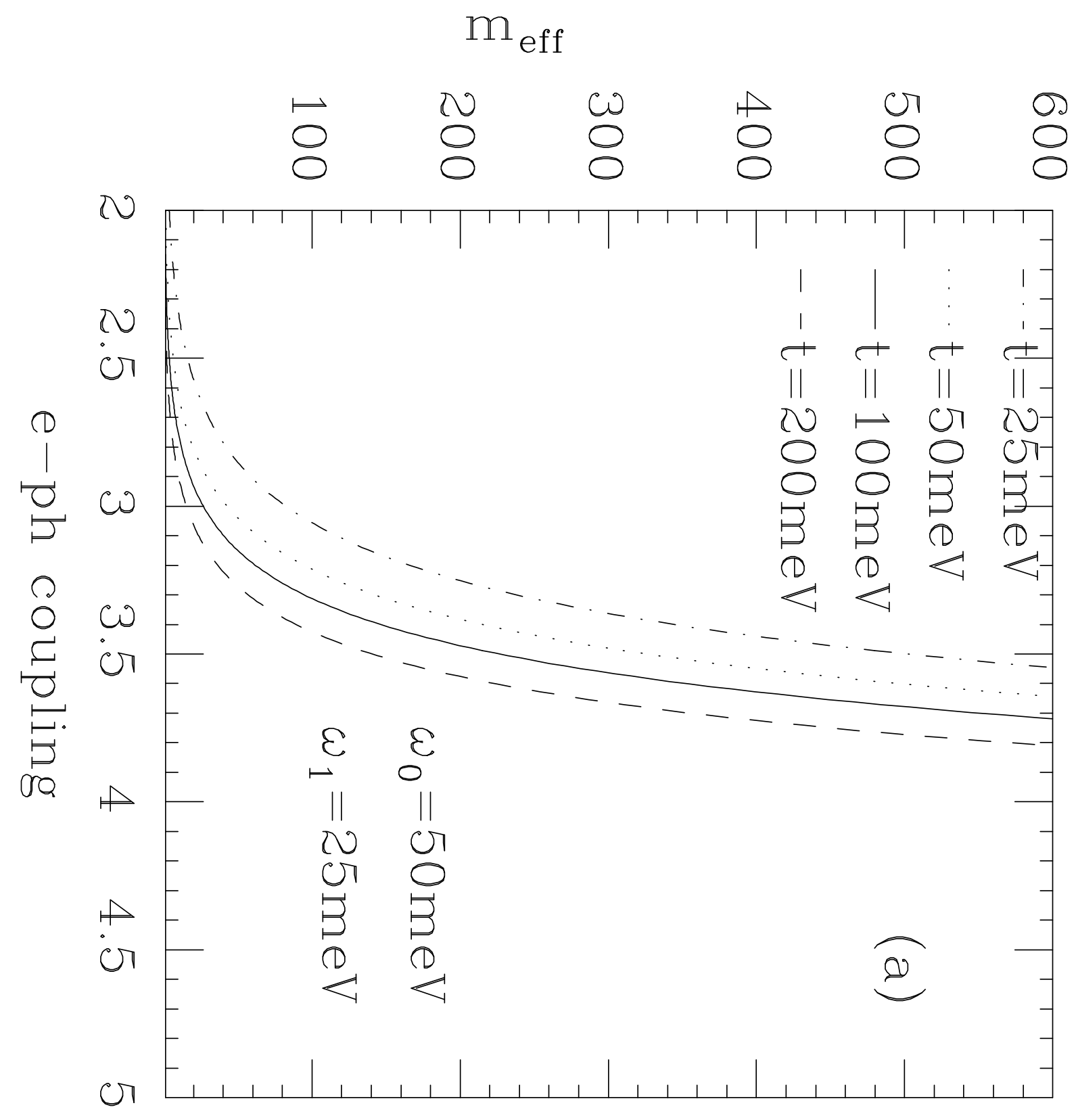




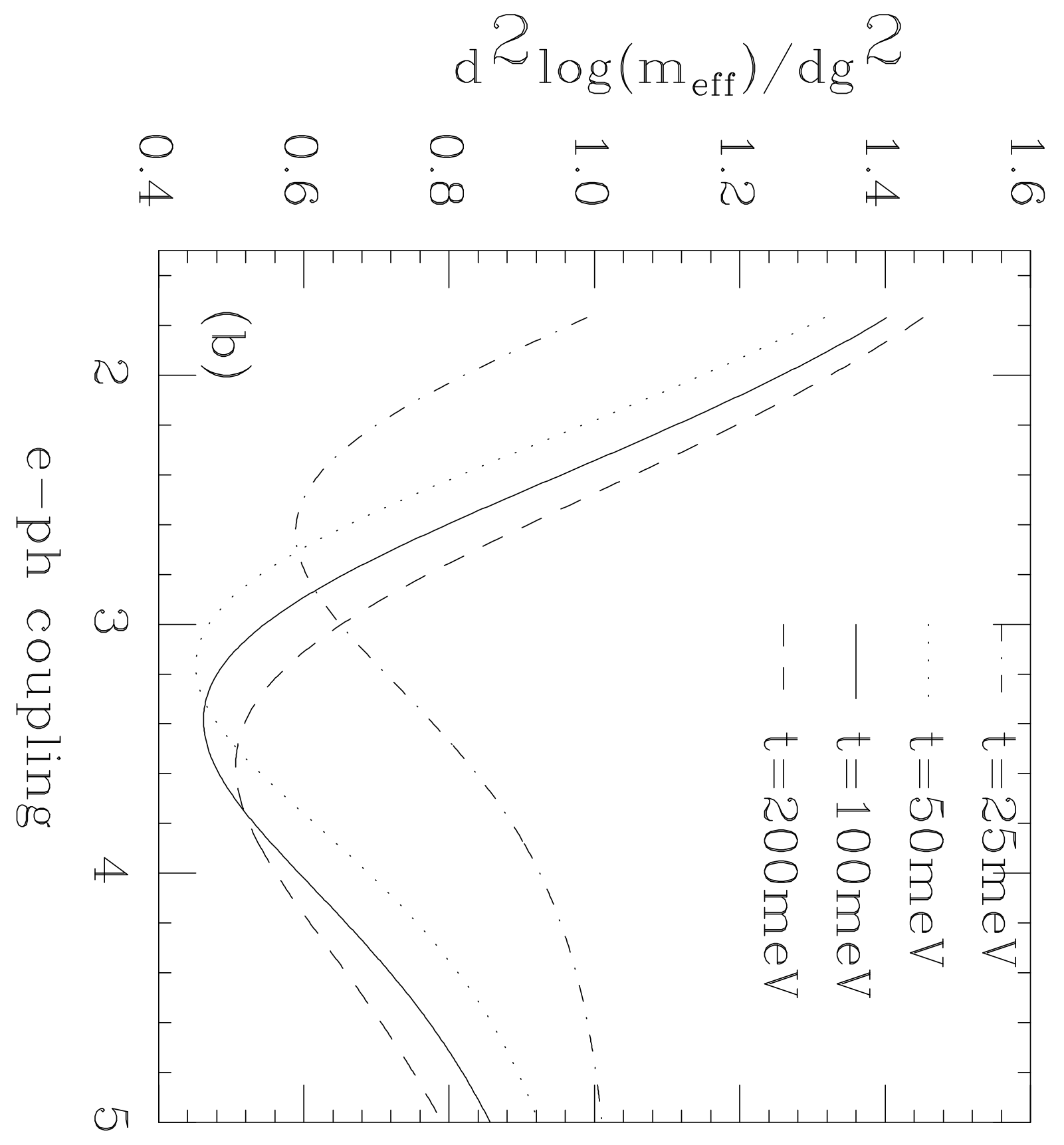




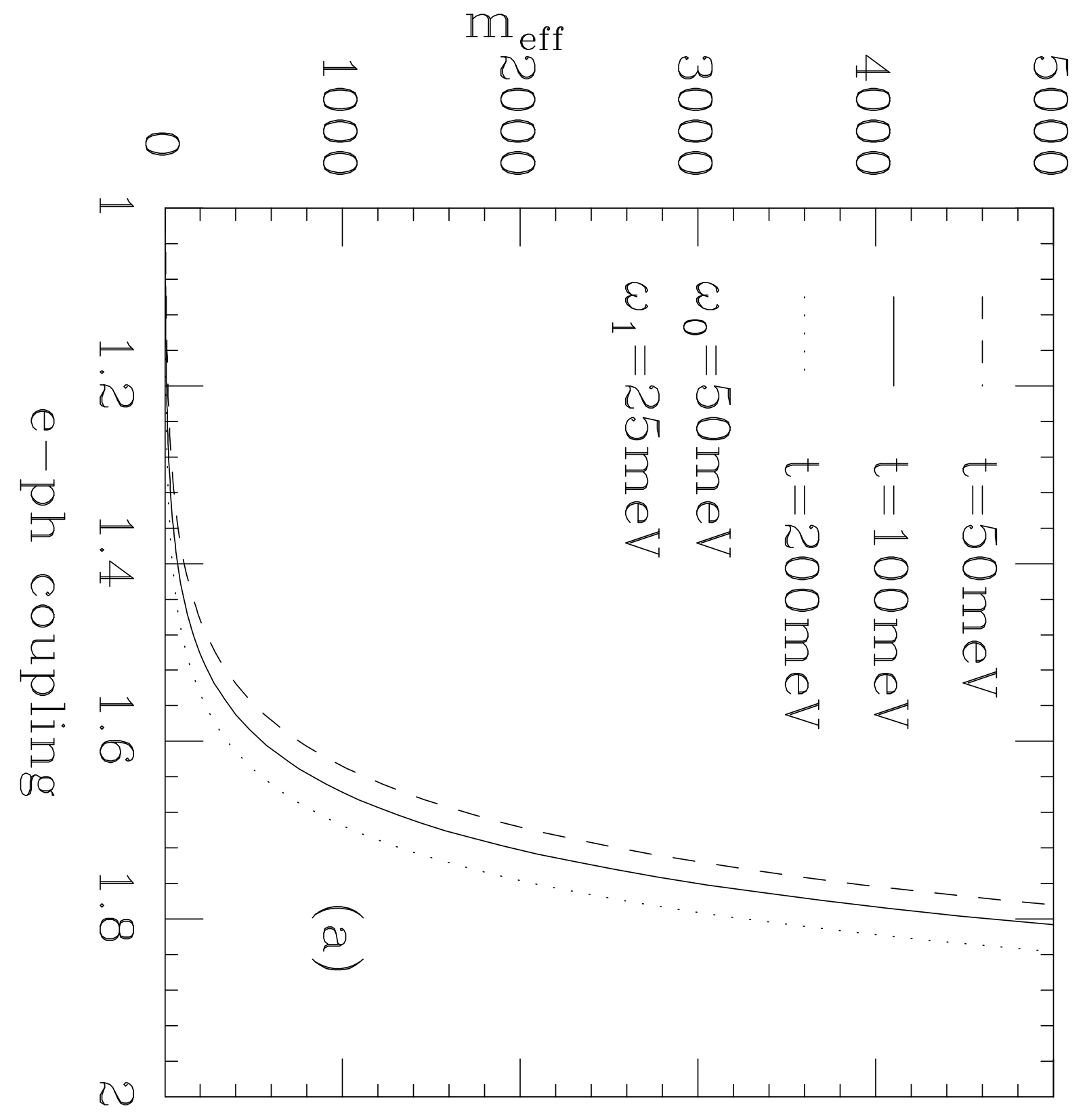




$$
\mathrm{d}^{2} \log \left(\mathrm{m}_{\mathrm{eff}}\right) / \mathrm{dg}{ }^{2}
$$

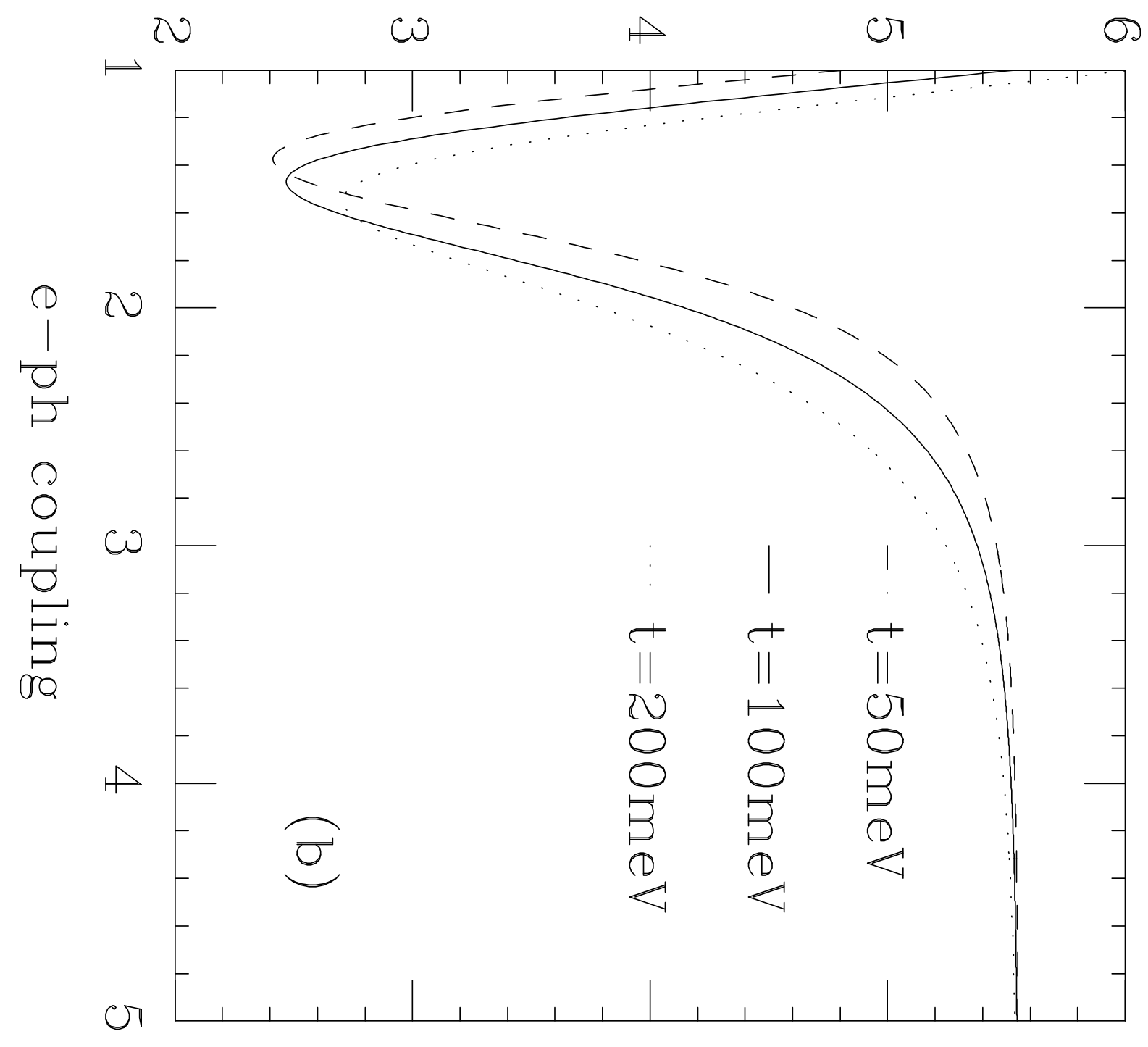

